\title{
What brain plasticity reveals about the nature of consciousness: commentary
}

\author{
Chris D. Frith ${ }^{1,2 *}$ \\ 1 Center for Functional Integrative Neuroscience, Aarhus University, Aarhus, Denmark \\ 2 Wellcome Trust Centre for Neuroimaging, University College London, London, UK \\ *Correspondence: cfrith@fil.ion.ucl.ac.uk
}

\section{WHAT IS CONSCIOUSNESS?}

Consciousness continues to be an illdefined concept, so I shall start by discussing how the term is used in this special issue. As discussed in Overgaard and Overgaard (2010), there is an important distinction between level of consciousness and content of consciousness. Level of consciousness refers to a dimension that varies from coma at one extreme, through sleep and, at the other extreme, alert wakefulness. Philosophers call this creature consciousness since it applies to the whole creature (Rosenthal, 2009). Level of consciousness is of particular relevance to the studies of patients in persistent vegetative state discussed by Laureys and colleagues (Demertzi et al., 2011). However, most of the contributions to this special issue are concerned with the content of consciousness.

Alert wakefulness is characterized by consciousness of specific mental states. The states that we are aware of are the contents of consciousness. Philosophers call this state consciousness. This is somewhat confusing, given that, when people talk about altered states of consciousness, they are usually referring to different levels of consciousness rather than different contents of consciousness. So I will continue to use the terms level and contents of consciousness.

In studies of the neural correlates of consciousness there is great interest in contrasting the neural activity associated with stimuli that influence the contents of consciousness with the neural activity associated with the same stimuli when they affect behavior in the absence of any change in the content of consciousness (Frith et al., 1999). When a stimulus elicits neural activity and affects behavior it does not necessarily follow that we are aware of that stimulus.

A certain minimal level of consciousness is necessary for there to be any contents of consciousness, but the level of consciousness does not determine what the contents of consciousness will be. As is demonstrated in this special issue, brain plasticity has an important role in determining the contents of consciousness.

There is also a reflective aspect of consciousness which is modeled by Cleeremans (2011) and which Allen and Williams (2011) suggest may be uniquely human. Are conscious mental states thoughts about thoughts? Is consciousness by its very nature reflective? There is clearly a relationship between this aspect of consciousness and metacognition. I shall return to consideration of this relationship at the end of this introduction.

\section{CERTAIN NEURAL STRUCTURES ARE NECESSARY, BUT NOT SUFFICIENT FOR CONSCIOUSNESS}

It is well established that brain lesions can alter the contents of consciousness. To take just one example, lesions to the extra-striate cortex can eliminate awareness of color (achromatopsia, Zeki, 1990). Brain stimulation can also generate the contents of consciousness. For example, electrical stimulation of extrastriate cortex can generate hallucinations of various visual features including color (Lee et al., 2000). This has lead to the idea of essential nodes for the specific conscious contents (e.g., V4 for color, Zeki and Bartels, 1999). In accord with this idea, Silvanto and Rees (2011) conclude that, in the absence of primary visual cortex, humans seem to have extremely limited capacity for visual awareness (but see Ffytche and Zeki, 2011).

However, these essential nodes are clearly not sufficient for consciousness. If the level of consciousness is too low, as in coma, then experience does not occur even though the essential nodes are intact. Laureys and colleagues (Demertzi et al., 2011) suggest that the lack of awareness in such cases is due to the loss of certain kinds of long-range connectivity in the brain. Similar effects may be produced by anesthesia in which long-ranged connectivity is lost temporarily (Alkire et al., 2008).

\section{BRAIN PLASTICITY PROVIDES IMPORTANT CLUES FOR UNDERSTANDING THE NATURE OF CONSCIOUSNESS AND ITS RELATION TO THE BRAIN}

The theme of this special issue is the observation that the loss of awareness associated with brain damage is frequently reversed. Unilateral spatial neglect, for example, is a disorder of consciousness associated with stroke from which recovery can be quite rapid (Cappa and Perani, 2010). In some cases it may be that brain tissue has been temporarily been deactivated, and subsequently recovers. However, in the many cases where brain tissue has been permanently damaged, we have to ask how such recovery is possible. The doctrine of essential nodes would suggest that, if the node has been destroyed, recovery should not be possible.

Mogensen (2011) presents an excellent discussion of this problem. Does recovery depend upon the growth of new connections? Does the patient develop new cognitive strategies? One important conclusion is that the brain activity supporting recovery need not be in the same location as that originally supporting the experience. This observation supports two conclusions: (1) Conscious experience (qualia) can be reacquired through some sort of learning process. (2) The nature of the experience (qualia) is not solely determined by the nature/location of the brain activity supporting it. This second conclusion is dramatically illustrated in the studies from Kupers et al. (2011) in which congenitally blind subjects were trained to "see" using a tactile stimulator. This technique involves turning the $2 \mathrm{D}$ images picked up by a video camera into a corresponding pattern of $2 \mathrm{D}$ tactile stimulation applied to the tongue. After being trained to recognize simple patterns with this stimulator, brain imaging revealed that performance of the task elicited activity in visual cortex. In addition 
transcranial magnetic stimulation applied to visual cortex lead to the experience tactile qualia. This is evidence against the idea, know as cortical dominance (Hurley and Noë, 2003), that qualia are determined by the cortical location of the associated brain activity. But what then is the property of nervous activity that determines the difference in the experience of the different senses?

\section{NEW QUALIA CAN BE LEARNED}

However, it is not only after brain damage that qualia can be relearned and even learned for the first time. There are many examples of learning in the normal case. For example, between 6 and 12 months infants lose awareness of speech sound distinctions not present in their native language. With sufficiently early intervention this loss of awareness can be reversed, but interestingly only through direct interaction with a speaker, rather than passive exposure to audio or video-tapes (Kuhl et al., 2003).

Normal subjects can also learn to become conscious of stimuli previously outside awareness. Schwiedrzik et al. (2009) used meta-contrast masking to achieve chance performance in the detection of stimuli. After 5 days of training sensitivity was significantly increased and subjects reported awareness of the stimuli. Gottfried and his colleagues (Li et al., 2008) exposed volunteers to odor molecules (rose oxide and 2-butanol) that exist in two mirror image forms (enantiomers). At the beginning of the experiment the participants were entirely unable to smell any differences between the two mirror image forms, as is the case for most people. After only seven trials (for each odor) of standard Pavlovian conditioning, participants exhibited fear responses to the odor associated with shock and not to the other form, indicating that they now could distinguish between the mirror image odors. A further perceptual experiment showed that participants could now consciously detect the difference in smell.

In these examples, it seems likely that the potential to make perceptual distinctions was already present in the brain and that training revealed and enhanced this ability. For example, given the nature of the human eye we would not expect training to lead to awareness of infrared or ultraviolet light.
But the potential for awareness can also be artificially modified. Genetic manipulation in both mice and monkeys can alter the perception of color. Male squirrel monkeys are normally dichromats, but, even as adults, can be turned into trichromats through insertion of the missing opsin gene (Mancuso et al., 2009).

\section{BUT HOW DO WE COMPARE OUALIA?}

The observation that new qualia can be acquired, whether through learning or gene therapy, reminds us of a fundamental problem in consciousness research. How can we compare qualia from one person to another? Or within the same person at different times? As Overgaard and Mogensen (2011) ask, when a brain damaged patient recovers an awareness that had been lost, how can we know whether it is the same as the awareness that was present before the brain damage? If this recovered awareness is instantiated by activity in a different brain region and depends upon a different cognitive strategy it might well be different. Are there methods for determining whether two seemingly identical conscious states are actually different?

We have long known that people do have different sensory experiences. An obvious example is color blindness. The presence of the receptors necessary for color vision is under genetic control and some people have only two receptors instead of three, leading to different forms of color blindness (dichromacy), depending on which particular pigment is missing. The visual qualia of the color blind is clearly different, but trichromats still have some idea of what color blindness is like. It has now been found that some women have more than three retinal photopigment genes. These women also perceive significantly more color appearances than men or women with the usual three photopigment genes (Jameson et al., 2001). In this case the discovery of the biological difference led to the identification of the difference in the experience of color that can be explored empirically by asking subjects to make fine color discriminations.

Another example concerns individual differences in the spatial extent of primary visual cortex (V1). People with larger V1 are more susceptible to size illusions (Schwarzkopf et al., 2011). Having identified this biological difference we now explore the idea that these people have a subtly different experience of space.
Probably the most striking success in comparing qualia across people is Bartoshuk's et al. (2004) demonstration of the existence of supertasters. These are people who experience the sense of taste with a far greater intensity than average. This discovery depended upon the development of scales for subjective experience that do not eliminate individual differences. There is still much work to be done in developing scales for quantifying subjective experience (see, for example, Sandberg et al., 2010), but it is clearly possible for such comparisons to be made.

\section{HOW CAN WE LEARN NEW QUALIA?}

Outside the laboratory human beings spend a lot of time in discussing their experiences. We enjoy telling each other what something was like. When we share experiences with others in this way, we can learn about two kinds of things. We can learn that other people have different experiences from ourselves. However, by pooling our experiences we can also get a better estimate of that the world is like, since, most of the time, two heads are better than one (Bahrami et al., 2010).

In order to pool our experiences we need to down play our differences and take the best features from each experience. Since successful joint action (as well as joint perception) depends upon such pooling, this may be why we are so often unaware of subtle, but consistent differences in experience. The implication is that, as a result of sharing experiences, our qualia may shift toward that of the person we are sharing with. I predict that the greatest shift will occur in the least expert member of the group. So I find most plausible the suggestion from Allen and Williams (2011), that we learn new qualia by interacting with others. This seems to be the case, for example, with activities like wine tasting (Smith, 2007). But for sharing our experiences we have to introspect upon and communicate our experience. This requirement emphasizes the reflective aspect of consciousness that is probably uniquely human. Reflecting upon our own experience is an example of metacognition, that is thinking about our thoughts.

There are considerable advantages for concentrating on this aspect of consciousness since metacognition is more precisely defined. Furthermore powerful techniques 
are now available for the quantification of metacognition (e.g., Galvin et al., 2003) and such measures have been applied to show that disruption of activity in dorsolateral prefrontal can change meta-cognitive sensitivity without altering discrimination performance (Rounis et al., 2010). Cleeremans (2011) uses the concept of metacognition to develop a computational model of how a brain can learn to be conscious by constructing a theory of its own behavior.

For me, a particularly interesting idea for further exploration is that this process of learning to be conscious of new things (i.e., to acquire new qualia) critically depends upon social interactions. In the various examples I mentioned above the learning of new qualia depended upon feedback from a teacher. To learn to experience the difference between the mirror image smell molecules required the experimenter to signal the distinction. More particularly, the American babies only learned to make the distinctions involved in Mandarin Chinese phonology through direct interaction with a speaker (Kuhl et al., 2003).

\section{CONCLUSION}

This special issue on the relevance of brain plasticity to the understanding of consciousness reminds us that consciousness, and the qualia that make up that consciousness, are not static. The contents of consciousness are constantly changing and developing through our experiences and especially through our sharing of experiences with others. Such change and development does not cease after brain damage. Indeed it is the dynamic relationship between brain and consciousness that enables the recovery of lost experience.

\section{REFERENCES}

Alkire, M. T., Hudetz, A. G., and Tononi, G. (2008). Consciousness and anesthesia. Science 322, 876-880. Allen, M., and Williams, G. (2011). Consciousness, plasticity, and connectomics: the role of intersubjectivity in human cognition. Front. Psychol.2:20. doi: 10.3389/ fpsyg.2011.00020

Bahrami, B., Olsen, K., Latham, P. E., Roepstorff, A., Rees, G., and Frith, C. D. (2010). Optimally interacting minds. Science 329, 1081-1085.

Bartoshuk, L. M., Duffy, V. B., Green, B. G., Hoffman, H. J., Ko, C. W., Lucchina, L. A., Marks, L. E., Snyder, D. J., and Weiffenbach, J. M. (2004). Valid across-group comparisons with labeled scales: the gLMS versus magnitude matching. Physiol. Behav. 82, 109-114.

Cappa, S. F., and Perani, D. (2010). Imaging studies of recovery from unilateral neglect. Exp. Brain Res. 206, 237-241.

Cleeremans, A. (2011). The radical plasticity thesis: how the brain learns to be conscious. Front. Psychol. 2:86. doi: 10.3389/fpsyg.2011.00086

Demertzi, A., Schnakers, C., Soddu, A., Bruno, M.-A. 1., Gosseries, O., Vanhaudenhuyse, A., and Laureys, S. (2011). Neural plasticity lessons from disorders of consciousness. Front. Psychol. 1:245. doi: 10.3389/ fpsyg.2010.00245

Ffytche, D. H., and Zeki, S. (2011). The primary visual cortex, and feedback to it, are not necessary for conscious vision. Brain 134(Pt 1), 247-257.

Frith, C., Perry, R., and Lumer, E. (1999). The neural correlates of conscious experience: an experimental framework. Trends Cogn. Sci. (Regul. Ed.) 3, 105-114.

Galvin, S. J., Podd, J.V., Drga, V., and Whitmore, J. (2003). Type 2 tasks in the theory of signal detectability: discrimination between correct and incorrect decisions. Psychon. Bull. Rev. 10, 843-876.

Hurley, S., and Noë,A. (2003). Neural plasticity and consciousness. Biol. Philos. 18, 131-168.

Jameson, K. A., Highnote, S. M., and Wasserman, L. M. (2001). Richer color experience in observers with multiple photopigment opsin genes. Psychon. Bull. Rev. 8, 244-261.

Kuhl, P. K., Tsao, F. M., and Liu, H. M. (2003). Foreignlanguage experience in infancy: effects of short-term exposure and social interaction on phonetic learning. Proc. Natl. Acad. Sci. U.S.A. 100, 9096-9101.

Kupers, R., Pietrini, P., Ricciardi, E., and Ptito, M. (2011). The nature of consciousness in the visuallydeprived brain. Front. Psychol. 2:19. doi: 10.3389/ fpsyg.2011.00019

Lee, H. W., Hong, S. B., Seo, D. W., Tae, W. S., and Hong, S. C. (2000). Mapping of functional organization in human visual cortex: electrical cortical stimulation. Neurology 54, 849-854.

Li, W., Howard, J. D., Parrish, T. B., and Gottfried, J. A. (2008). Aversive learning enhances perceptual and cortical discrimination of indiscriminable odor cues. Science 319, 1842-1845.

Mancuso, K., Hauswirth, W. W., Li, Q., Connor, T. B., Kuchenbecker, J.A., Mauck, M. C., Neitz, J., and Neitz,
M. (2009). Gene therapy for red-green colour blindness in adult primates. Nature 461, 784-787.

Mogensen, J. (2011). Reorganization of the injured brain: implications for studies of the neural substrate of cognition. Front. Psychol. 2:7. doi: 10.3389/ fpsyg.2011.00007

Overgaard, M., and Mogensen, J. (2011). A framework for the study of multiple realizations: the importance of levels of analysis. Front. Psychol. 2:79. doi: 10.3389/ fpsyg.2011.00079

Overgaard, M., and Overgaard, R. (2010). Neural correlates of contents and levels of consciousness. Front. Psychol. 1:164. doi: 10.3389/fpsyg.2010.00164

Rosenthal, D. (2009). "Concepts and definitions of consciousness," in Encyclopedia of Consciousness, ed. P.W. Banks (Amsterdam: Elsevier), 157-169.

Rounis, E., Maniscalco, B., Rothwell, J. C., Passingham, R. E., and Lau, H. (2010). Theta-burst transcranial magnetic stimulation to the prefrontal cortex impairs metacognitive visual awareness. Cogn. Neurosci. 1, 165-175.

Sandberg, K., Timmermans, B., Overgaard, M., and Cleeremans, A. (2010). Measuring consciousness: is one measure better than the other? Conscious. Cogn.19, 1069-1078.

Schwarzkopf, D. S., Song, C., and Rees, G. (2011). The surface area of human $\mathrm{V} 1$ predicts the subjective experience of object size. Nat. Neurosci. 14, 28-30.

Schwiedrzik, C. M., Singer, W., and Melloni, L. (2009). Sensitivity and perceptual awareness increase with practice in metacontrast masking. J. Vis. 9, 18 11-18.

Silvanto, J., and Rees, G. (2011). What does neural plasticity tell us about role of primary visual cortex (V1) in visual awareness? Front. Psychol. 2:6. doi: 10.3389/ fpsyg.2011.00006

Smith, B. C. (ed.). (2007). Questions of Taste: The Philosophy of Wine. Oxford: Signal Books.

Zeki, S. (1990). A century of cerebral achromatopsia. Brain 113(Pt 6), 1721-1777.

Zeki, S., and Bartels, A. (1999). Toward a theory of visual consciousness. Conscious. Cogn. 8, 225-259.

Received:08 March 2011; accepted:26April 2011; published online: 11 May 2011.

Citation: Frith CD (2011) What brain plasticity reveals about the nature of consciousness: commentary. Front. Psychology 2:87. doi: 10.3389/fpsyg.2011.00087

This article was submitted to Frontiers in Consciousness Research, a specialty of Frontiers in Psychology.

Copyright (C) 2011 Frith. This is an open-access article subject to a non-exclusive license between the authors and Frontiers Media SA, which permits use, distribution and reproduction in other forums, provided the original authors and source are credited and other Frontiers conditions are complied with. 